\title{
Molecular profiling in breast cancer-ready for clinical routine?
}

\author{
Kristina A. Tendl · Zsuzsanna Bago-Horvath
}

Received: 16 December 2019 / Accepted: 12 January 2020 / Published online: 6 February 2020

(C) The Author(s) 2020

Summary The herald of genomic testing opened novel diagnostic and therapeutic possibilities for many tumor entities. For breast cancer, molecular profiling has become an integral part of disease management on multiple levels. Genetic testing allows for the identification of hereditary cancer syndromes in patients with a family history of malignancies and contributes to the successful prevention of breast cancer. In early breast cancer, several prospective randomized trials demonstrated the prognostic significance of commercially available mRNA-based gene expression analyses, which now have become part of standard of care in the adjuvant setting. In advanced breast cancer, testing for targetable mutations ensures personalized cancer treatment. Poly-ADPribose polymerase (PARP) inhibitors provide the first targeted alternative for patients with BRCA 1/2-associated breast cancer. In advanced breast cancer of luminal type, the detection of Phosphatidylinositol4,5-Bisphosphate 3-Kinase Catalytic Subunit Alpha (PIK3CA) mutations provides a novel treatment option with alpelisib, a PIK3CA inhibitor. Further targetable mutations include NTRK3 in rare cases of secretory breast carcinoma and human epidermal growth factor receptor 2 (HER2). Recent data support the importance of the analysis of circulating tumor cells and cell-free DNA. These "liquid biopsies" open novel possibilities of molecular profiling. However, clinical benefit of such analyses remains to be confirmed.

Keywords Hereditary breast cancer - Genetic testing • Multigenomic assays · Targetable mutations · Liquid biopsy

\section{Genetic testing in hereditary cancer syndromes}

Results from the prospective EMBRACA study demonstrated that affected patients with relevant $B R C A 1$ and $B R C A 2$ mutations carry a lifetime cumulative risk of $60 \%$ and $55 \%$ to develop breast cancer, respectively [1]. Besides BRCA 1/2, mutations in several other genes that are associated with an increased risk of developing breast cancer have been identified. These include p53, PTEN, CDH1, and PALB2 [2]. Affected patients may be entitled to participate in intensified high-risk screening programs and prophylactic surgery. These developments have contributed to an increased demand for genetic testing. Recent technical advances and cost efficacy of next generation sequencing (NGS) aid the large-scale implementation of high-throughput molecular diagnostics to identify patients at risk. NGS methods provide through the simultaneous testing of multiple genes robust and timely results. Especially the detection of large genomic rearrangements (LGR) is warranted by NGS methods in comparison to Sanger sequencing [2]. However, for most of these genes, variants of unknown clinical significance exist that should be considered in the applied guidelines. The interpretation of results should also take family history of cancer into consideration. Recent challenges include interpreting complex sequencing data, whereas some results may require confirmation by Sanger sequencing. Another difficulty is posed by the testing of tumor samples for BRCA 1/2 mutations, as a germline mutation cannot be confirmed from these samples and certain types of large genomic rearrangements might be missed. 


\section{Transcriptome-based multigenomic assays in early luminal breast cancer}

Immunohistochemically defined prognostic and predictive markers, such as estrogen- (ER) and progesterone receptor (PR), human epidermal growth factor receptor 2 (HER2) and the proliferation marker Ki67 still govern the therapy recommendation in early breast cancer in many cases [3]. However, the discovery that these characteristics are mirrored by welldefined patterns in gene expression allowed a further precision of prognostication and facilitated the development of transcriptome-based multigenomic assays that correlate well with pathological markers but still provide independent prognostic and in some instances, predictive information [4, 5]. Especially in ER-positive luminal-type early breast cancer, additional prognostic information is often required to provide patients with a valid and effective therapy recommendation. Whereas endocrine therapy is offered for most patients with luminal breast cancer, only a subset of patients derives clinical benefit from adjuvant chemotherapy. Whereas patients with lowproliferative luminal A disease do not benefit from the addition of adjuvant chemotherapy to endocrine therapy, adjuvant chemotherapy should be offered to patients with more aggressive and highly proliferative luminal B disease [6]. Although luminal A and B disease might be identified by immunohistochemical markers, especially by the proliferation marker Ki67, determination is subjected to significant inter- and intraobserver variability that impair objective and reproducible measurements [7]. Quantitative analysis of the RNA expression of several relevant genes of luminal tumors might capture tumor biology in a more accurate and reproducible way. Commercially available multigenomic assays are able to discriminate luminal A from luminal B biology and provide patients with a clinically valid therapy recommendation.

Most frequently applied multigenomic platforms include the 21-gene Oncotype Dx recurrence score [8], the 70-gene MammaPrint assay [9], the 12-gene Endopredict assay [10] and the PAM50 risk of recurrence score [11]. Multigenomic assays of the first generation, namely Oncotype Dx and MammaPrint assess mostly tumor proliferation, while tests of the second generation, Endopredict and PAM50 also measure genes of ER signaling. Whereas all of these tests were retrospectively validated on tumor material collected from prospective randomized clinical trials [12], the clinical validity of Oncotype Dx and MammaPrint was also confirmed in prospective randomized trials.

The TAILORx study included women with ER-positive, node-negative breast cancer. Treatment choice was stratified by the Oncotype Dx recurrence score [13]. Patients with recurrence scores between 11 and 25 were randomized to receive endocrine therapy alone versus adjuvant chemo- and endocrine ther- apy. Study results demonstrated that these patients derived no benefit from the addition of chemotherapy. However, for women younger than 50 years and tumors with recurrence scores between 16 and 25, chemotherapy was associated with an improved survival. Further studies are needed to confirm these results and whether this benefit is in fact caused by ovarian function suppression in premenopausal patients by chemotherapy.

Clinical utility of the MammaPrint assay was confirmed by the prospective randomized MINDACT trial [14]. In this phase III study, 6693 patients with early stage breast cancer were enrolled. Genomic risk was determined by MammaPrint and clinical risk was estimated by a modified version of Adjuvant! Online. Patients with discrepant genomic and clinical risk were randomized to receive endocrine therapy alone versus adjuvant chemo- and endocrine therapy. Neither patients with low clinical risk/high genomic risk nor those with high clinical risk/low genomic risk derived significant benefit from adjuvant chemotherapy. Interestingly, molecular subtyping of these tumors by MammaPrint/BluePrint identified more luminal A tumors than centrally reviewed pathological subtyping by ER, PR, HER2 and Ki67, with comparable clinical outcome [15]. Further studies should clarify if selected patients with low-risk HER2-positive luminal tumors can be spared adjuvant chemotherapy.

Several studies demonstrated that assays of the second generation, PAM50 and Endopredict are also able to predict late tumor recurrence beyond 5 years [16, 17]. Analysis of the involved genes in the Endopredict assay revealed that mainly genes associated with ER signaling contributed to the additional prognostic information regarding late relapses. These results suggest that such tumors might respond well to an extended endocrine therapy regime. Prospective studies to confirm these hypotheses are warranted.

Although sufficient data support the use of multigenomic assays to guide therapy decisions in early breast cancer, the concordance between different platforms is moderate at best, as shown by the results of the OPTIMA prelim trial [18]. In this study, risk stratification was performed by several different platforms such as Oncotype Dx, PAM50, MammaPrint, MammaTyper and IHC4. The five assays displayed modest agreement, as only $39.4 \%$ of tumors were uniformly assigned to the high versus low/intermediate risk group. Although previous studies suggest that the prognostic information provided by multigenomic assays is comparable, test results of different platforms might be unsettling for the individual patients. However, increasing implementation of multigenomic assays is expected to decrease the rate of adjuvant chemotherapy for the treatment of early luminal breast cancer. 


\section{Molecular profiling of advanced breast cancer}

Despite the technological advances, little data supports the routine use of large-scale genomic testing of tumors in advanced breast cancer. Results of the first clinical trials have been disappointing, as in spite of the presence of targetable mutations in a majority of enrolled patients, only $11 \%$ had actually been treated according to their molecular tumor profile [19]. However, for selected mutations, evidence from clinical trials supports the use of genomic profiling. PIK3CA mutations represent one of the most frequent genetic aberrations in luminal breast cancer and were shown to be associated with a favorable prognosis. However, PIK3CA-mutated HER2-positive breast cancer was demonstrated to be associated with worse prognosis [20]. The frequency of these mutations and the biological relevance of the pathway render PIK3CA a promising therapy target. However, first clinical trials using broad-spectrum pan-PIK3CA inhibitors, such as buparlisib demonstrated a relatively high risk of therapy-related adverse events (hyperglycemia, diarrhea, rash, transaminitis, mood disorders), that limit therapy efficacy due to frequent discontinuation of use [21]. In contrast, isoform-specific inhibitors can overcome these limitations and reach a broader therapeutic range.

Alpelisib is a p110 $\alpha$-isoform specific oral PIK3CA inhibitor. Results from the SOLAR I trial point out that tumors carrying PIK3CA mutations benefit from treatment with Alpelisib in combination with fulvestrant [22]. Clonal analyses revealed that PIK3CA mutations remain fairly constant between primary tumor and metastasis, meaning that testing of the primary tumor is feasible for therapy indication.

For several other mutations, tumor heterogeneity and genomic evolution in the course of tumor progression have been proven. Mutations of Estrogen Sensing Receptor 1 (ESR1) are shown to emerge in metastases compared to primary tumors and seem to contribute to resistance to endocrine therapy [23]. Whether testing for ESR1 mutation will be part of future routine molecular profiling protocols remains to be determined.

Approaches of synthetic lethality facilitated the development of PARP inhibitors for the treatment of BRCA mutated breast cancer. To date, two substances, olaparib and talazoparib have been approved by the FDA for the treatment of metastatic breast cancer in patients with germline $B R C A$ mutations, based on results of the OlympiAD and EMBRACA trials [1, 24]. Therefore, genetic testing of all patients with metastatic breast cancer should be considered as germline BRCA mutations have been found in approximately $5 \%$ of breast cancers.

A novel NTRK inhibitor, larotrectinib has shown activity against advanced secretory breast carcinoma, a rare special subtype of TNBC, which harbor an ETV6-NTRK3 gene fusion [25].
Patients with HER2 and HER3 mutations in the absence of HER2 amplification might be promising candidates for a targeted therapy with the kinase inhibitor neratinib [26].

Besides the above-mentioned genes, several large scale genomic test panels as well as tests for quantifying the overall mutational burden for breast cancer exist; however, evidence for clinical utility of these panels is controversial.

\section{ctDNA analysis and liquid biopsy}

Current research is exploring the prognostic and predictive role of circulating tumor cells (CTC) and circulating cell-free circulating DNA (ctDNA) from blood plasma as well as tumor-derived exosomes, which were also shown to mirror molecular tumor characteristics $[27,28]$. Mutations found in these liquid biopsies might reflect intratumoral heterogeneity, mutational shifts, epigenetic changes and clonal evolution in a more accurate way than samples from the primary tumor or isolated metastases [29]. Dynamic changes in the mutations detected might facilitate early detection of the development of resistance mechanisms and point to innovative approaches of personalized cancer therapy. Driver mutations, i.e. of TP53, PIK3CA and ESR1 can be detected in circulating tumor cells (CTC) as well as using ctDNA and have been shown to possess prognostic value and in some cases, these mutations were predictive of therapy resistance [30-33]. Liquid biopsies are likely to become an additional diagnostic tool that could aid personalized cancer therapy.

\section{Take home message}

- In hereditary breast cancer, in addition to BRCA 1/2, further gene mutations can be identified by NGS that are associated with an increased lifetime risk of developing breast cancer

- mRNA-based multigenomic assays are useful in aiding adjuvant therapy decisions

- Detection of targetable mutations of BRCA 1/2, PIK3CA and NTRK pose novel indications for biological therapies in advanced breast cancer

- Liquid biopsies that analyze circulating tumor cells, circulating cell-free DNA and tumor cell exosomes open new diagnostic and therapeutic possibilities that account for tumor heterogeneity in a more adequate way

Funding Open access funding provided by Medical University of Vienna.

Conflict of interest K.A. Tendl reports travel support from Roche, MSD and Novartis, outside the submitted work. Z. Bago-Horvath reports personal fees from Novartis, Biomedica and Roche and travel support from Roche, outside the submitted work. 
Open Access This article is licensed under a Creative Commons Attribution 4.0 International License, which permits use, sharing, adaptation, distribution and reproduction in any medium or format, as long as you give appropriate credit to the original author(s) and the source, provide a link to the Creative Commons licence, and indicate if changes were made. The images or other third party material in this article are included in the article's Creative Commons licence, unless indicated otherwise in a credit line to the material. If material is not included in the article's Creative Commons licence and your intended use is not permitted by statutory regulation or exceeds the permitted use, you will need to obtain permission directly from the copyright holder. To view a copy of this licence, visit http://creativecommons.org/licenses/by/4.0/.

\section{References}

1. Litton JK, Rugo HS, Ettl J, et al. Talazoparib in patients with advanced breast cancer and a germline BRCA mutation. NEngl J Med. 2018;379(8):753-63.

2. WallaceAJ. New challengesfor BRCA testing: a viewfrom the diagnostic laboratory. Eur J Hum Genet. 2016;24(1):10-8.

3. Curigliano G, Burstein HJ, Winer EP, et al. De-escalating and escalating treatments for early-stage breast cancer: the St. Gallen international expert consensus conference on the primary therapy of early breast cancer 2017 . Ann Oncol. 2017;28(8):1700-12.

4. Perou CM, Sørlie T, Eisen MB, et al. Molecular portraits of human breast tumours. Nature. 2000;406(6797):747-52.

5. Sørlie T, Perou CM, Tibshirani R, et al. Gene expression patterns of breast carcinomas distinguish tumor subclasses with clinical implications. Proc Natl Acad Sci U S A. 2001;98(19):10869-74.

6. Coates AS, Winer EP, Goldhirsch A, et al. Tailoring therapies-improving the management of early breast cancer: St. Gallen international expert consensus on the primary therapy of early breast cancer 2015 . Ann Oncol. 2015;26(8):1533-46.

7. VargaZ, DieboldJ, Dommann-ScherrerC, etal. Howreliable is Ki-67 immunohistochemistry in grade 2 breast carcinomas? A QA study of the Swiss working group of breast- and gynecopathologists. PLoS One. 2012;7(5):e37379.

8. Paik S, Shak S, Tang G, et al. A multigene assay to predict recurrence of tamoxifen-treated, node-negative breast cancer. NEngl J Med. 2004;351:2817-26.

9. van de Vijver MJ, He YD, van't Veer LJ, et al. A geneexpression signature as a predictor of survival in breast cancer. NEngl J Med. 2002;347:1999-2009.

10. Filipits M, Rudas M, Jakesz R, et al. A new molecular predictor of distant recurrence in ER-positive, HER2negative breast cancer adds independent information to conventional clinical risk factors. Clin Cancer Res. 2011;17(18):6012-20.

11. Wallden B, Storhoff J, Nielsen T, et al. Development and verification of the PAM50-based Prosigna breast cancer gene signature assay. BMCMed Genomics. 2015;8:54.

12. Azim HA Jr, Michiels S, Zagouri F, et al. Utility of prognostic genomic tests in breast cancer practice: the IMPAKT 2012 working group consensus statement. Ann Oncol. 2013;24(3):647-54.

13. Sparano JA, Gray RJ, Makower DF, et al. Adjuvant chemotherapy guided by a 21-gene expression assay in breast cancer. NEngl J Med. 2018;379:111-21.

14. Cardoso F, van't Veer LJ, Bogaerts J, et al. MINDACT Investigators. 70-gene signature as an aid to treatment decisions in early-stage breast cancer. $\mathrm{N}$ Engl J Med. 2016;375:717-29.
15. Viale G, Slaets L, de Snoo FA, et al. Discordant assessment of tumor biomarkers by histopathological and molecular assays in the EORTC randomized controlled 10041/BIG 03-04 MINDACT trial breast cancer : intratumoral heterogeneity and DCIS or normal tissue components are unlikely to be the cause of discordance. Breast Cancer Res Treat. 2016;155(3):463-9.

16. Filipits M, Nielsen TO, Rudas M, et al. The PAM50 riskof-recurrence score predicts risk for late distant recurrence after endocrine therapy in postmenopausal women with endocrine-responsive early breast cancer. Clin Cancer Res. 2014;20(5):1298-305.

17. Dubsky P, Brase JC, Jakesz R, et al. The EndoPredict score provides prognostic information on late distant metastases in ER+/HER2-breast cancer patients. Br J Cancer. 2013;109(12):2959-64.

18. Bartlett JM, Bayani J, Marshall A, et al. Comparing breast cancer multiparameter tests in the OPTIMA prelim trial: no test is more equal than the others. J Natl Cancer Inst. 2016;108(9):djw50. https://doi.org/10.1093/jnci/djw050.

19. Santa-Maria CA, Kruse M, Raska P, et al. Impact of tissue-based genomic profiling on clinical decision making in the management of patients with metastatic breast cancer at academic centers. Breast Cancer Res Treat. 2017;166(1):179-84.

20. Verret B, Cortes J, Bachelot T, Andre F, Arnedos M. Efficacy of PI3K inhibitors in advanced breast cancer. Ann Oncol. 2019;30(10):x12-x20.

21. Baselga J, Im SA, Iwata $\mathrm{H}$, et al. Buparlisib plus fulvestrant versus placebo plus fulvestrant in postmenopausal, hormone receptor-positive, HER2-negative, advanced breast cancer (BELLE-2): a randomised, double-blind, placebocontrolled, phase 3 trial. Lancet Oncol. 2017;18(7):904-16.

22. AndréF, Ciruelos E, Rubovszky G, etal. Alpelisibfor PIK3CAmutated, hormone receptor-positive advanced breast cancer. NEngl J Med. 2019;380(20):1929-40.

23. Robinson DR, Wu YM, Vats P, et al. Activating ESR1 mutations in hormone-resistant metastatic breast cancer. NatGenet. 2013;45(12):1446-51.

24. Robson M, Im SA, Senkus E, et al. Olaparib for metastatic breast cancer in patients with a germline BRCA mutation. NEngl J Med. 2017;377(6):523-33.

25. Landman Y, Ilouze M, Wein S, et al. Rapid response to larotrectinib (LOXO-101) in an adult chemotherapy-naive patients with advanced triple-negative secretory breast cancer expressing ETV6-NTRK3 fusion. Clin Breast Cancer. 2018;18(3):e267-e70.

26. Hyman DM, Piha-Paul SA, Won H, et al. HER kinase inhibition in patients with HER2- and HER3-mutant cancers. Nature. 2018;554(7691):189-94.

27. Litton JK, Burstein HJ, Turner NC. Molecular testing in breastcancer. AmSocClin OncolEducBook. 2019;39:el-e7.

28. O'Leary B, Hrebien S, Morden JP, et al. Early circulating tumor DNA dynamics and clonal selection with palbociclib and fulvestrant for breast cancer. Nat Commun. 2018;9(1):896.

29. Hench IB, Hench J, Tolnay M. Liquid biopsy in clinical management of breast, lung, and colorectal cancer. Front Med (Lausanne). 2018;5:9.

30. Madic J, Kiialainen A, Bidard F-C, et al. Circulating tumor DNA and circulating tumor cells in metastatic triple negative breast cancer patients: ctDNA and CTC in metastatic triple negative breast cancer. Int J Cancer. 2015;136:2158-65.

31. Higgins MJ, JelovacD, Barnathan E, etal. Detection of tumor PIK3CA status in metastatic breast cancer using peripheral 
blood. Clin Cancer Res. 2012;18:3462-9. https://doi.org/ 10.1158/1078-0432.

32. Takeshita T, Yamamoto Y, Yamamoto-Ibusuki M, et al. Prognostic role of PIK3CA mutations of cell-free DNA in early-stage triple negative breast cancer. Cancer Sci. 2015;106:1582-9.

33. Chu D, Paoletti C, Gersch C, et al. ESR1 mutations in circulating plasma tumor DNA from metastatic breast cancer patients. Clin Cancer Res. 2016;22:993-9.

Publisher's Note Springer Nature remains neutral with regard to jurisdictional claims in published maps and institutional affiliations.
- For latest news from international oncology congresses see: http://www.springermedizin.at/ memo-inoncology 